\title{
Piceatannol Suppresses the Proliferation and Induced Apoptosis of Osteosarcoma Cells Through PI3K/AKT/mTOR Pathway
}

This article was published in the following Dove Press journal: Cancer Management and Research

\section{Bin Wang ${ }^{\prime}$ \\ Jingyu $\mathrm{Li}^{2}$}

'Third Department of Orthopaedics, Shengjing Hospital of China Medical University, Shenyang, Liaoning, People's Republic of China; ${ }^{2}$ Department of Ultrasonography, Shengjing Hospital of China Medical University, Shenyang, Liaoning, People's Republic of China
Correspondence: Jingyu $\mathrm{Li}$

Department of Ultrasonography, Shengjing Hospital of China Medical

University, No. 36, Sanhao Street, Heping

District, Shenyang, Liaoning II0000,

People's Republic of China

Tel + 86-24-966I5333II

Email jingy_lijyu@।63.com
Background: Piceatannol (PIC) is confirmed to inhibit the proliferation of various tumors, but its role in osteosarcoma still remains unknown. This study investigated the function of PIC in osteosarcoma, aiming to provide a research basis for osteosarcoma therapy.

Methods: Human osteosarcoma cells in this study were, respectively, treated with PIC at various concentrations $(0,20,40,80 \mu \mathrm{mol} / \mathrm{L})$, PI3K/AKT/mTOR pathway inhibitor (LY294002), or pathway activator (740Y-P) plus PIC. Osteosarcoma cells were subcutaneously injected into nude mice to establish xenograft models, and PIC was intraperitoneally injected into models. The activity, proliferation, apoptosis and cell cycle of treated cells were determined by MTT test, colony formation assay or a flow cytometry. The expressions of $\mathrm{PI} 3 \mathrm{~K} / \mathrm{AKT} / \mathrm{mTOR}$ pathway-related proteins in cells and tumor tissues were measured by Western blot (WB) analysis.

Results: PIC (40 and $80 \mu \mathrm{mol} / \mathrm{L}$ ) and LY294002 availably suppressed activity and proliferation and induced apoptosis of osteosarcoma cells. PIC observably increased the number of cells retarded in G2 phase, but decreased the cell percentages in G1 and S phases. Conversely, 740Y-P reversed the effects of PIC on osteosarcoma cells, which promoted cell activity and proliferation and restrained apoptosis. In xenograft models, the volume and weight of the tumors treated by PIC were visibly alleviated than those untreated. The PI3K/ AKT/mTOR pathway was prominently inhibited in PIC-treated osteosarcoma cells and tumor tissues.

Conclusion: PIC suppresses the proliferation and induces apoptosis of osteosarcoma cells through regulating $\mathrm{PI} 3 \mathrm{~K} / \mathrm{AKT} / \mathrm{mTOR}$ pathway, which is expected to be the therapeutic of osteosarcoma.

Keywords: osteosarcoma, piceatannol, proliferation, apoptosis, PI3K/AKT/mTOR pathway

\section{Introduction}

Osteosarcoma is one of the usual primary malignant bone tumors in children and adolescents, which has the characteristics of early lung metastasis and high recurrence rate. ${ }^{1,2}$ With the continuous development of surgery and chemotherapy, the 5-year survival rate of osteosarcoma patients has increased to $60-70 \%$, but there are approximately $30-50 \%$ of patients still have localized recurrence. ${ }^{3-5}$ Currently, there is no effective treatment for recurrent and metastatic osteosarcoma in clinical practice. ${ }^{6}$ Although high-dose neoadjuvant chemotherapy drugs such as methotrexate, doxorubicin, cisplatin and ifosfamide are of positive significance in the treatment of osteosarcoma, high doses of drugs have great side 
effects, so there are still some limitations. ${ }^{7}$ In recent years, most of the antineoplastic drugs developed are not aimed at osteosarcoma, and the research and development of new clinical drugs are relatively confined, which may be due to the fact that the disease is rare in a wide range of clinical practice. Therefore, finding a new effective and safe treatment method to improve the therapeutic effect of osteosarcoma is the key issue that needs to be solved.

Piceatannol (PIC) is a chemical structure similar to resveratrol compounds, widely found in white tea, passion fruit, grapes, and Japanese knotweed and other plants. ${ }^{8}$ Some scholars have found that PIC has the biological activities of anti-oxidation, scavenging free radicals, regulating immunity and anti-inflammation, as well as its antioxidation effect is stronger than that of resveratrol. ${ }^{9,10}$ In previous studies, it has been attested that PIC can inhibit the activity of enzymes that promote the development of tumor cells, and thus interfere with the growth of tumor cells, so as to play an anti-tumor role. ${ }^{11}$ In addition, the antioxidant effect of PIC can alleviate the damage of excessive reactive oxygen species and free radical to DNA, and reduce the incidence of cancer. ${ }^{12}$ In clinical application, Du et al $^{13}$ pointed out that PIC could restrict the growth of melanoma cells and induce apoptosis. In addition, Hsieh et al ${ }^{14}$ reported that PIC could regulate the cycle, proliferation and apoptosis of prostate cancer cells through the AKT/mTOR pathway. Mori reported that PIC could significantly abrogate the proliferation of MC3T3-E1 cells, which were cultured in conditioned media from mouse OC. ${ }^{15}$ Chang reported that PIC exhibited a significant induction of differentiation in MG-63 cells. ${ }^{16}$ Recently, Li found that PIC could suppress AKT activation, promote $\mathrm{G} 2 / \mathrm{M}$ phase arrest and induce mitochondrial apoptosis, moreover, it could increase Cisplatin effect in OS cells. ${ }^{17}$ Nevertheless, the probable mechanisms were still unclear.

In this study, human osteosarcoma cells were treated with PIC, PI3K/AKT/mTOR signaling pathway inhibitor or activator, and their effects on cell cycle, proliferation, apoptosis and related proteins expressions were analyzed. Moreover, we also established in vivo nude mice xenograft models to further verify the effect of PIC on the growth of tumors. The purpose of this study was to explore the effect of PIC on osteosarcoma cells and the related mechanism, so as to provide a potential treatment scheme for the therapeutics of osteosarcoma and ameliorate the treatment outcome.

\section{Materials and Methods}

\section{Ethics Statement}

All animal experiments involved in this study were authorized by the Ethic Committee of Shengjing Hospital of China Medical University, and followed the guidelines of Animal Care and Institutional Ethical in China.

\section{Cell Culture}

Human osteosarcoma cell lines (U2OS and MG-63) and human normal osteoblasts cells (hFOB1.19) were purchased from the Cell Bank of Type Culture Collection of Chinese Academy of Sciences (Shanghai, China). Cells were cultured in Dulbecco's Modified Eagle Medium (DMEM; Hyclone, Logan, UT, USA) containing 1\% penicillin/streptomycin (Sigma-Aldrich, St. Louis, MO, USA) and $10 \%$ fetal bovine serum (FBS; Invitrogen, Carlsbad, California, USA). The cell culture was in a humidified condition with $5 \% \mathrm{CO}_{2}$ at $37^{\circ} \mathrm{C}$.

\section{Cell Treatment}

Human osteosarcoma cell lines (U2OS and MG-63) and human normal osteoblasts (hFOB1.19) were seeded in 96well culture plates at a density of $5 \times 10^{3}$ cells/well, and incubated for $24 \mathrm{~h}$. Then, all cells were treated with PIC (purity > 98\%; \#E-1255, Tauto Biotech, Shanghai, China) at various concentrations $(20,40,80 \mu \mathrm{mol} / \mathrm{L})$. Moreover, 10 $\mu \mathrm{mol} / \mathrm{L}$ of the $\mathrm{PI} 3 \mathrm{~K} / \mathrm{AKT} / \mathrm{mTOR}$ pathway inhibitor (LY294002; \#A8250, APExBIO, USA) was added into U2OS and MG-63 cells, while human osteosarcoma cell lines exposed to PIC $(80 \mu \mathrm{mol} / \mathrm{L})$ received the addition of PI3K/AKT/mTOR pathway activator (740Y-P; \#BCP16353, $15 \mu \mathrm{mol} / \mathrm{L}$, BioChemPartner, Shanghai, China). All treatments lasted $48 \mathrm{~h}$ and cells were collected for subsequent experiments. For comparison, untreated cells were served as controls.

\section{Cell Activity}

After different treatments, 3-(4,5-dimethythiazol-2-yl)2,5-diphenyltetrazolium bromide (MTT) assays were performed to measure the activity of human osteosarcoma cell lines and normal osteoblasts. Briefly, after treatment for $48 \mathrm{~h}$, $20 \mu \mathrm{L}$ MTT solution (Sigma-Aldrich, USA) was added into cells for another $4 \mathrm{~h}$ at $37^{\circ} \mathrm{C}$. The absorbance was measured with ELX-800 Biotek plate reader (Winooski, USA) at $490 \mathrm{~nm}$. 


\section{Colony Formation Assay}

After treatment, human osteosarcoma cell lines were shifted into 12-well plates (100 cells/well) and cultured for 10 days. Then, the colonies were fixed with $10 \%$ formaldehyde for $10 \mathrm{~min}$ and stained with $1.0 \%$ crystal violet for $10 \mathrm{~min}$. The number of cells was photographed and calculated under the Olympus CKX41 microscope (Tokyo, Japan).

\section{Cell Apoptosis}

The apoptosis rate of human osteosarcoma cell lines was confirmed with an annexin V-fluorescein isothiocyanate (FITC) kit (Beyotime, Shanghai, China). After treatment, a volume of $5 \mu \mathrm{L}$ Annexin V-FITC and $5 \mu \mathrm{L}$ of propidium iodide (PI) were added into human osteosarcoma cell lines in the dark for $5 \mathrm{~min}$. The apoptosis rate of cells was determined by FACSCaliburTM Flow Cytometer (BD Bioscience, USA) according to the manufacturer's instructions.

\section{Cell Cycle}

For cell cycle assay, PIC-treated human osteosarcoma cell lines were rinsed with phosphate buffer saline (PBS) solution, and fixed with $75 \%$ ethanol for $30 \mathrm{~min}$. Afterwards, cells were incubated with bovine pancreatic $2 \mu \mathrm{g} / \mathrm{mL}$ RNAase (Sigma) and PI solution $(10 \mu \mathrm{g} / \mathrm{mL}$, Invitrogen) at room temperature for $30 \mathrm{~min}$ each. The cell number at each cell cycle phase was examined by FACSCaliburTM Flow Cytometer (BD Bioscience, USA).

\section{Animal Experiment}

A total of 6 female BALB/c nude mice (6 weeks old) provided by Vital River Laboratory Animal Technology (Beijing, China) were feed in bioclean conditions with a $12 \mathrm{~h} \mathrm{light/dark}$ cycle. $1 \times 10^{6}$ of U2OS cells were subcutaneously injected into the mice to establish in vivo tumor models. When the volume of xenografts grew about $100 \mathrm{~mm}^{3}$, intraperitoneal injection of PIC $(20 \mathrm{mg} / \mathrm{kg} /$ day $)$ was performed on 3 cases of mice, and another 3 mice treated with normal saline were used as controls. The volume of tumor was detected by a caliper every 3 days until 21 days. On the last day, mice were sacrificed by overdosed anaesthetic, and the tumors were separated from them and then weighed.

\section{Western Blot (WB) Analysis}

The expressions of PI3K/AKT/mTOR pathway-related proteins in human osteosarcoma cell lines and mice tumor tissues were assessed by WB assays. For the detection, cells and tumor tissues were dissolved in RIPA buffer (Solarbio, Beijing, China) to extract the total protein. Bicinchoninic Protein Assay kit (BCA, Pierce, Rockford, IL, USA) was carried out to measure the concentration of extracted protein. Then, $50 \mu \mathrm{g}$ of the total protein was exposed to $10 \%$ sodium dodecyl sulfate-polyacrylamide gel electrophoresis (SDS-PAGE, Beyotime, Shanghai, China) and followed by shift onto polyvinylidene fluoride (PVDF) membranes. Subsequently, the membranes were sealed with 5\% skimmed milk for $2 \mathrm{~h}$, and co-incubated with the primary antibodies $(1: 1000$, Cell Signaling Technology, USA) at $4{ }^{\circ} \mathrm{C}$ overnight, including phosphorylated (p)-PI3K (\#4228), PI3K (\#4292), p-Akt (\#9271), Akt (\#9272), p-mTOR (\#2971), mTOR (\#2983), and $\beta$-actin (\#4967) was taken as the internal reference. The corresponding secondary antibody (\#7074) was added for $1 \mathrm{~h}$ at room temperature. The blots signals were developed by an enhanced chemiluminescence-detecting kit (Thermo Fisher, MA, USA).

\section{Statistical Analysis}

GraphPad Prism 8.0 software (GraphPad Software, Inc., La Jolla, CA, USA) was used for data analysis. The measurement data were presented as mean \pm standard deviation (SD). The comparison between two or more groups was performed by Student's $t$-test or one-way analysis of variance (ANOVA). All experiments were implemented in triplicate. $P<0.05$ was considered as statistically significant.

\section{Results}

\section{PIC Inhibited the Proliferation of Osteosarcoma Cells and Induced Apoptosis}

In MTT assays, it could be seen that PIC at different concentrations had no significant effect on the activity of normal osteoblasts, while PIC at concentrations of 40 and $80 \mu \mathrm{mol} / \mathrm{L}$ notably inhibited the activity of human osteosarcoma cell lines U2OS and MG-63 ( $P<0.01$; Figure 1A). Accordingly, colony formation assay illuminated that the proliferation ability of human osteosarcoma cell lines was prominently reduced under the PIC of 40 and $80 \mu \mathrm{mol} / \mathrm{L}$ $(P<0.001$; Figure 1B-E). Moreover, the results from flow cytometer showed that PIC of 40 and $80 \mu \mathrm{mol} / \mathrm{L}$ obviously elevated the apoptosis rate of human osteosarcoma cell lines $(P<0.001$; Figure $1 \mathrm{~F}-\mathrm{I})$. As for the cell cycle 
A

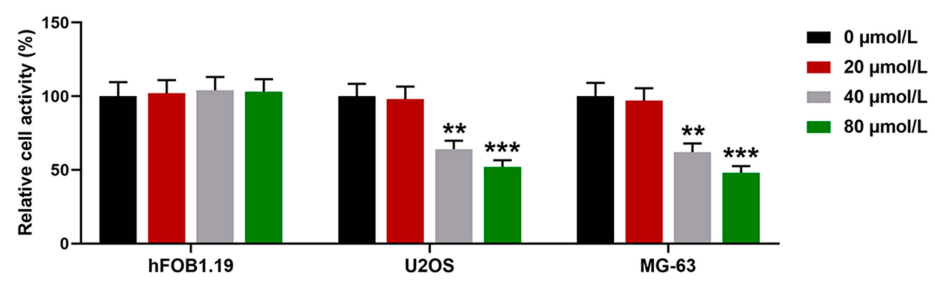

B
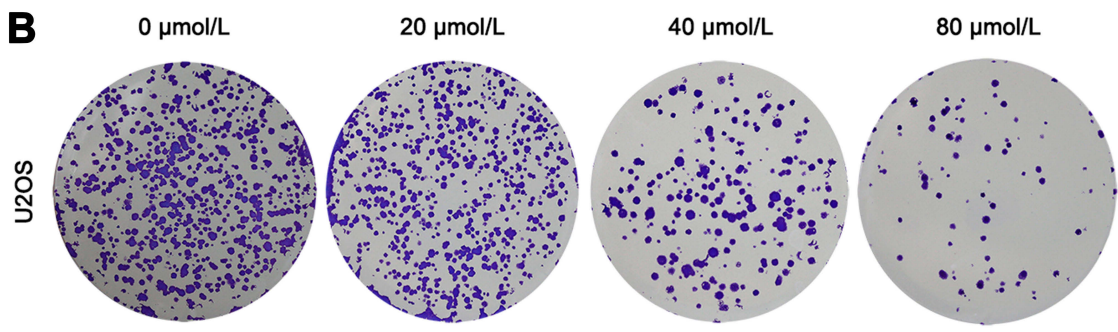

D $\quad 0 \mu \mathrm{mol} / \mathrm{L}$
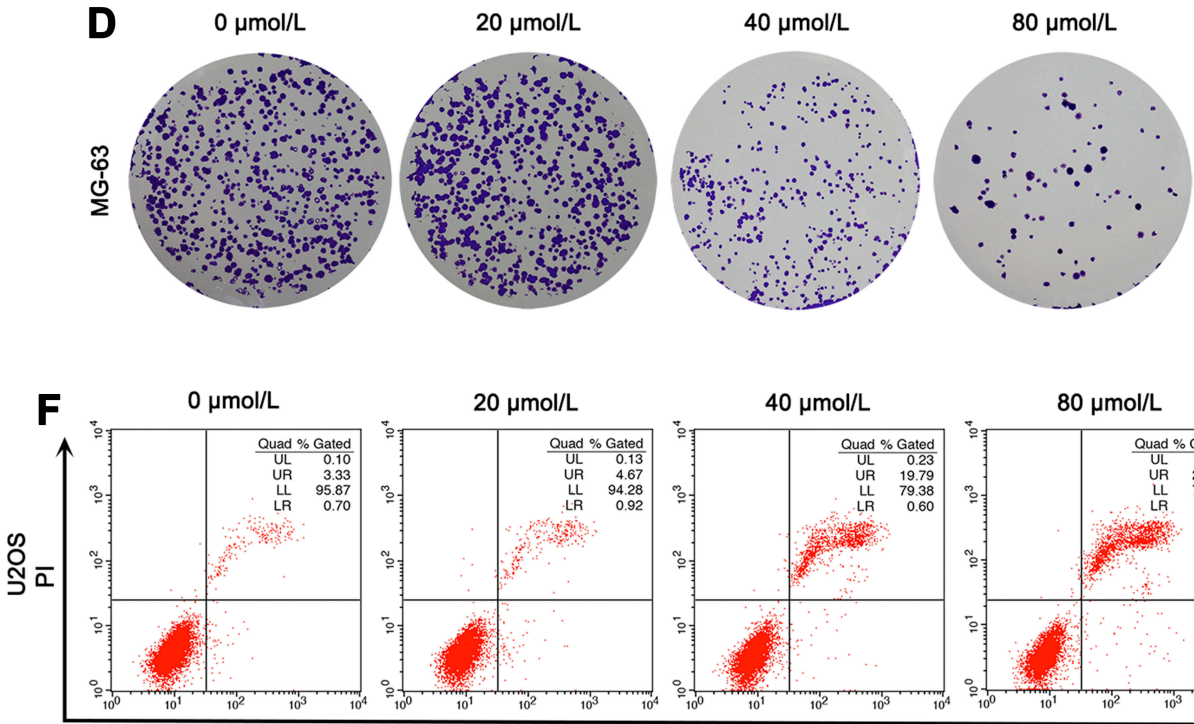

Aneexin-V-FITC
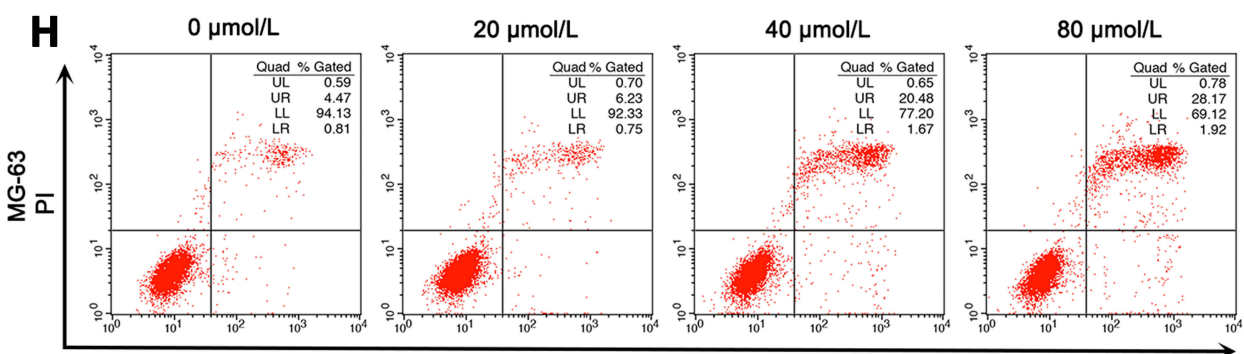

$80 \mu \mathrm{mol} / \mathrm{L}$

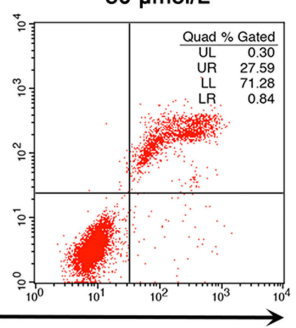

G

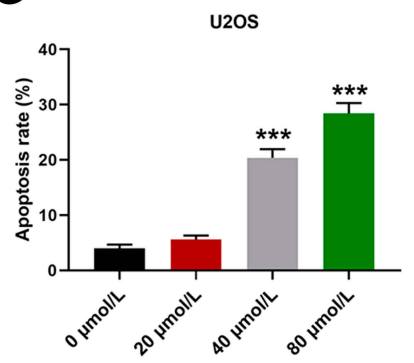

I

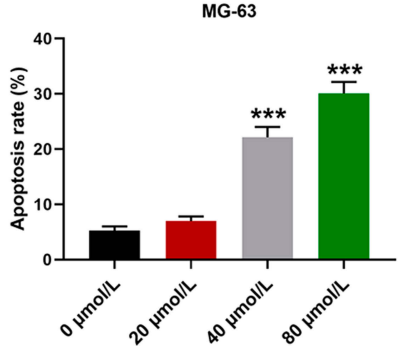

Figure I Piceatannol (PIC) inhibited the proliferation of osteosarcoma cells and induced apoptosis. In this figure, human osteosarcoma cell lines (U2OS and MG-63) and human normal osteoblasts (hFOBI.19) were treated with PIC at various concentrations $(0,20,40,80 \mu \mathrm{mol} / \mathrm{L})$. (A) The activities of cells after treatment were detected by 3-(4,5-dimethythiazol-2-yl)-2,5-diphenyltetrazolium bromide (MTT) assays. (B-E) The cloning images and quantitative analysis of treated human osteosarcoma cell lines (U2OS and MG-63) were measured by colony formation assay. (F-I) The apoptosis rates of human osteosarcoma cell lines (U2OS and MG-63) after treatment were determined though a flow analyzer. $* * P<0.01$, $* * * P<0.001$, vs $\mathrm{PIC}$ at $0 \mu \mathrm{mol} / \mathrm{L}$. All experiments were implemented in triplicate.

analysis, certain concentrations of PIC (40 and $80 \mu \mathrm{mol} / \mathrm{L}$ ) observably increased the number of cells retarded in G2 phase, but decreased the cell percentages in G1 and
$\mathrm{S}$ phases $(P<0.05$; Figure 2$)$. In general, PIC of $20 \mu \mathrm{mol} /$ $\mathrm{L}$ had no effective effect on the growth and apoptosis of osteosarcoma cells. 


\section{PIC Suppressed the Activation of PI3K/ AKT/mTOR Pathway}

In WB analysis, after treatment with PIC of $80 \mu \mathrm{mol} / \mathrm{L}$, the expression levels of $\mathrm{p}-\mathrm{PI} 3 \mathrm{~K}, \mathrm{p}$-Akt and p-mTOR in human osteosarcoma cell lines were all showed a decreasing trend, whereas the expressions of PI3K, Akt and mTOR were relatively stable in cells. As a result, the ratios of p-PI3K/PI3K (U2OS:16.36\%; MG-63: 22.69\%), p-Akt/ Akt (U2OS: 26.36\%; MG-63: 16.26\%) and p-mTOR/ mTOR (U2OS: 34.33\%; MG-63:15.58\%) were also significantly declining in U2OS and MG-63 cells by the effect of PIC at $80 \mu \mathrm{mol} / \mathrm{L}(P<0.001$; Figure 3$)$.

\section{$\mathrm{PI}$ KK/AKT/mTOR Pathway Agonist Rescued the Effects of $\mathrm{PCl}$ on Osteosarcoma Cells}

In order to probe into the mechanism of PIC on osteosarcoma, we used PI3K/AKT/mTOR pathway inhibitor (LY294002) and agonist (740Y-P) to treat human osteosarcoma cell lines. MTT results represented that, similar to the functions of PIC, LY294002 clearly restrained the activity of osteosarcoma cell lines, while 740Y-P reversed the inhibitory effect of PIC on the cell activity $(P<0.01$; Figure 4A and B). In colony formation assay, it could be viewed that both LY294002 and PIC significantly reduced the colony number of osteosarcoma cell lines, while 740Y-P promoted cell proliferation $(P<0.001$; Figure $4 \mathrm{C}-\mathrm{F})$. Corresponding to the above experimental findings, flow cytometry analysis revealed that LY294002 obviously accelerated the apoptosis of osteosarcoma cell lines, whereas 740Y-P effectively rescued the pro-death effect of PCI $(P<0.001$; Figure 4G-J).

\section{PIC Blocked the Growth of Osteosarcoma Tumors in Xenograft Models}

For in-depth exploration, this study established nude mice tumor-bearing models, and further analyzed the effects of PIC on animal. After intraperitoneal injection of PIC, the tumor volume showed a gradually increasing trend, but it was significantly slower than the untreated controls after 9 days of treatment $(P<0.05$; Figure 5A). On day 21 after injection, we isolated and weighed the tumors, and found that the size and weight of the tumors treated by PIC were
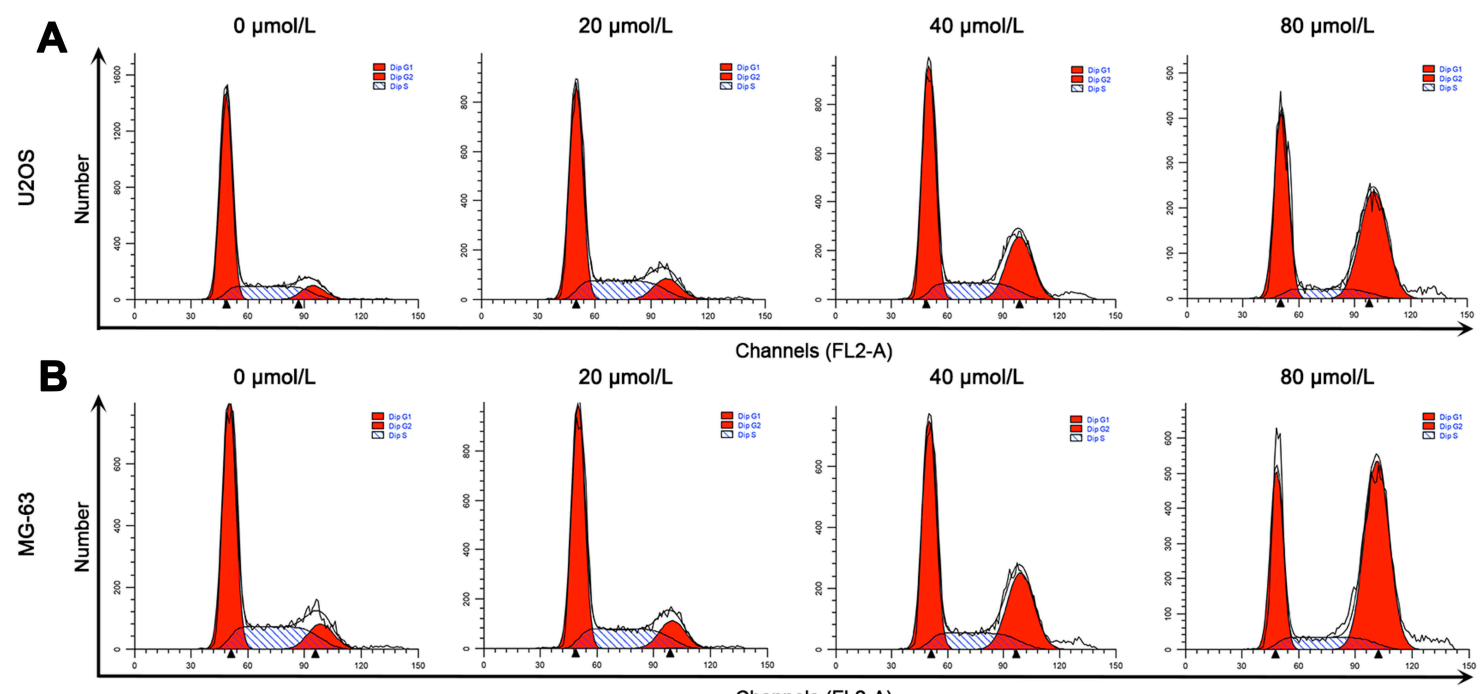

Channels (FL2-A) $\quad 40 \mu \mathrm{mol} / \mathrm{L}$
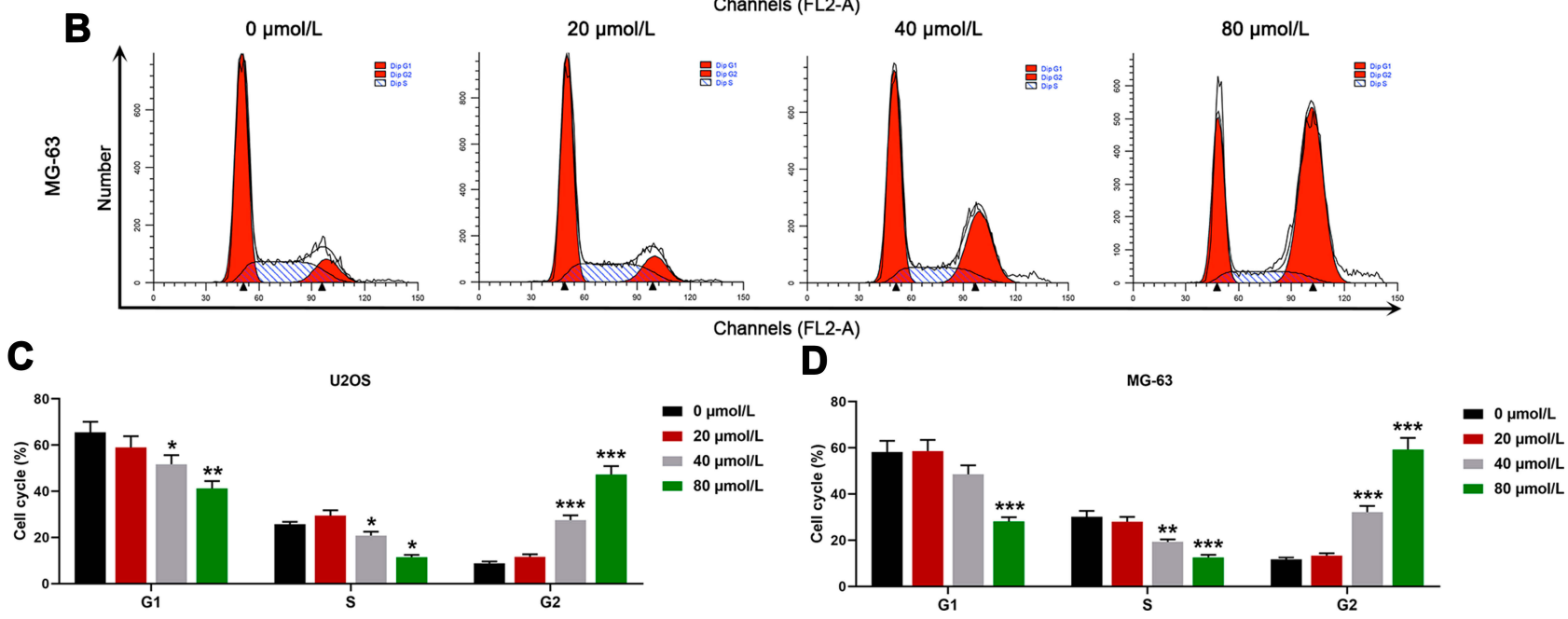

Figure 2 Piceatannol (PIC) increased the osteosarcoma cells remained in G2 cell cycle. In this figure, human osteosarcoma cell lines (U2OS and MG-63) were treated with $\mathrm{PIC}$ at various concentrations (0, 20, 40,80 $\mu \mathrm{mol} / \mathrm{L})$. (A-D) Cell cycle images and quantitative results of osteosarcoma cells (U2OS and MG-63) after treatment were obtained from flow cytometry analysis. $* P<0.05, * * P<0.0 \mathrm{I}, * * * P<0.00 \mathrm{I}$, vs $\mathrm{PIC}$ at $0 \mu \mathrm{mol} / \mathrm{L}$. All experiments were implemented in triplicate. 

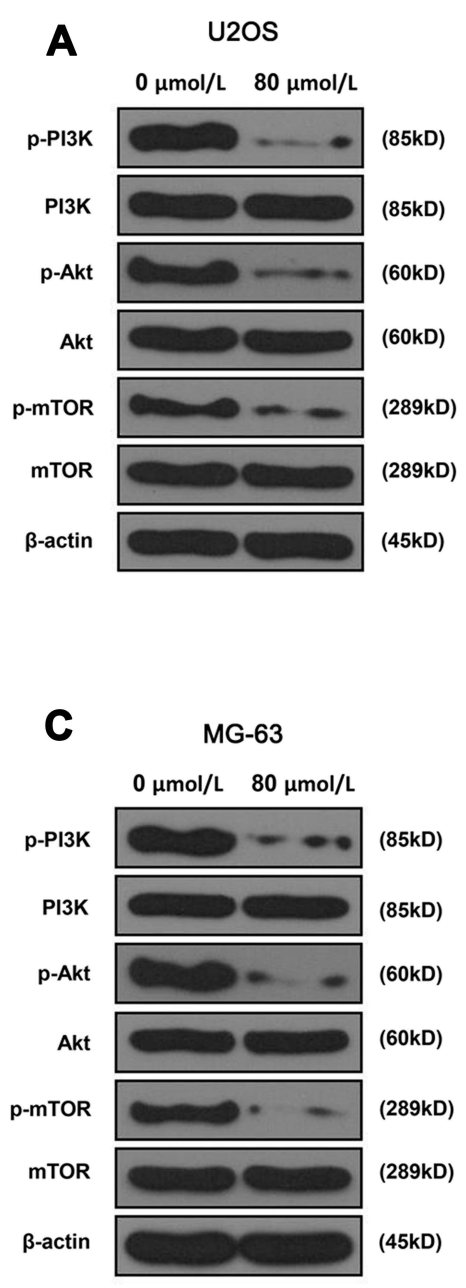

B

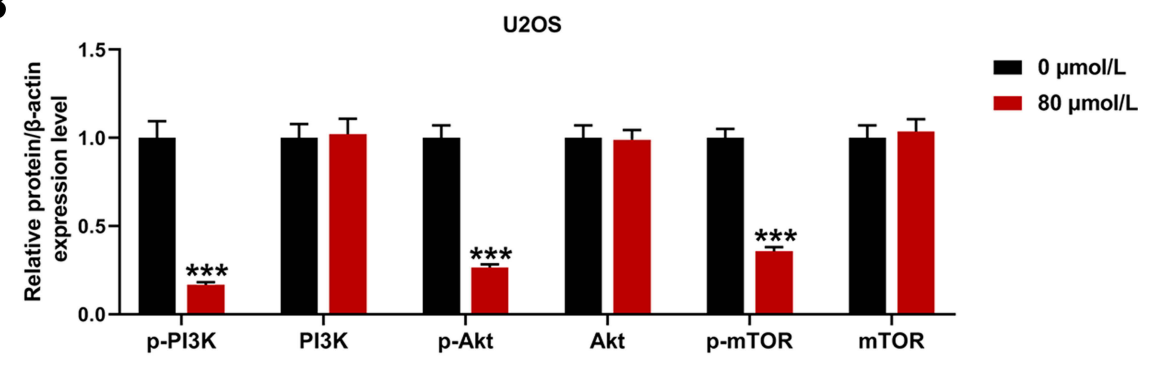

D

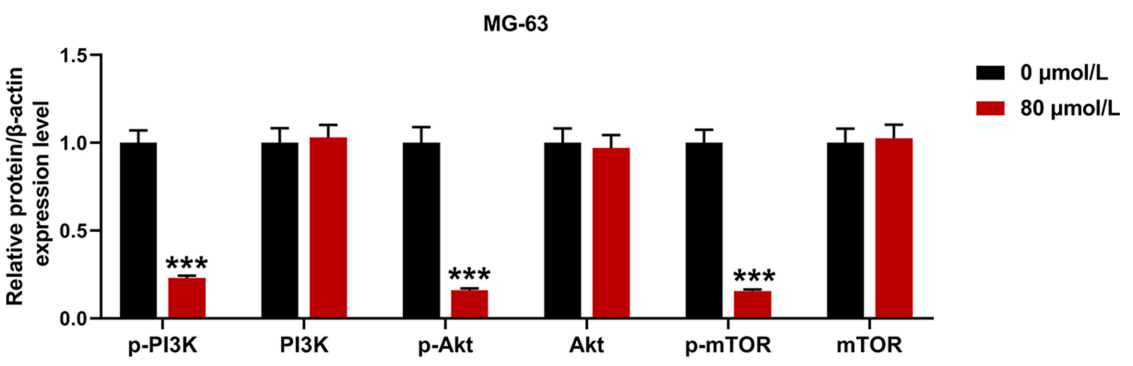

Figure 3 Piceatannol (PIC) suppressed the activation of PI3K/AKT/mTOR pathway in osteosarcoma cells. In this figure, human osteosarcoma cell lines (U2OS and MG-63) were treated with PIC at 0 or $80 \mu \mathrm{mol} / \mathrm{L}$. Western blot (WB) assay was implemented to measure the expression levels of phosphorylated (P) or not PI3K, Akt and mTOR in osteosarcoma cells U2OS (A-B) and MG-63 (C-D) after treatment. $* * * P<0.00 \mathrm{I}$, vs PIC at $0 \mu \mathrm{mol} / \mathrm{L}$. All experiments were implemented in triplicate.

visibly lower than those untreated $(P<0.001$; Figure 5B). In WB analysis, the expression levels of $\mathrm{p}-\mathrm{PI} 3 \mathrm{~K}$, p-Akt and $\mathrm{p}$-mTOR in PIC-treated tumor tissues were prominently depressed compared with controls, as well as the ratios of p-PI3K/PI3K (16.35\%), p-Akt/Akt (16.67\%) and $\mathrm{p}-\mathrm{mTOR} / \mathrm{mTOR}(14.02 \%)$ were also showed an evident downtrend $(P<0.001$; Figure 6$)$.

\section{Discussion}

PIC is a hydroxylation analogue of resveratrol, which has similar biological activities to resveratrol, such as antioxidant, anti-inflammatory and lipodieresis effects. ${ }^{18,19}$ Recently, the anti-tumor activity of PIC has gradually attracted the attention of clinical workers, and there are some scholars have reported that PIC could inhibit the invasion, migration and adhesion of breast cancer cells by blocking the activation of PI3K/AKT and NF- $\kappa$ B pathway. ${ }^{20}$ In the orthopedic system, Yan et $\mathrm{al}^{21}$ pointed out that PIC not only suppressed bone resorption and osteoclastogenesis but also accelerated apoptosis of mature osteoclasts, which might be beneficial to the therapy of correlative bone diseases. In a cell study, Wolter et $\mathrm{al}^{22}$ treated human colorectal cancer cell lines with PIC, and found that the drug reduced cell proliferation rate and inhibited cell cycle progression at $\mathrm{S}$ phase, in a time- and concentration-dependent manner. Thus, it can be seen that PIC plays a momentous role in the field of tumor, but its function in osteosarcoma still remains indistinct.

In this present study, we exposed human osteosarcoma cells to PIC at different concentrations, and revealed that relatively high concentrations of PIC (40, $80 \mu \mathrm{mol} / \mathrm{L})$ could suppress cell activity and proliferation while promoting apoptosis, and effectively facilitate the cell cycle into G2 phase. These results suggested that PIC can inhibit the proliferation of osteosarcoma cells and induce 


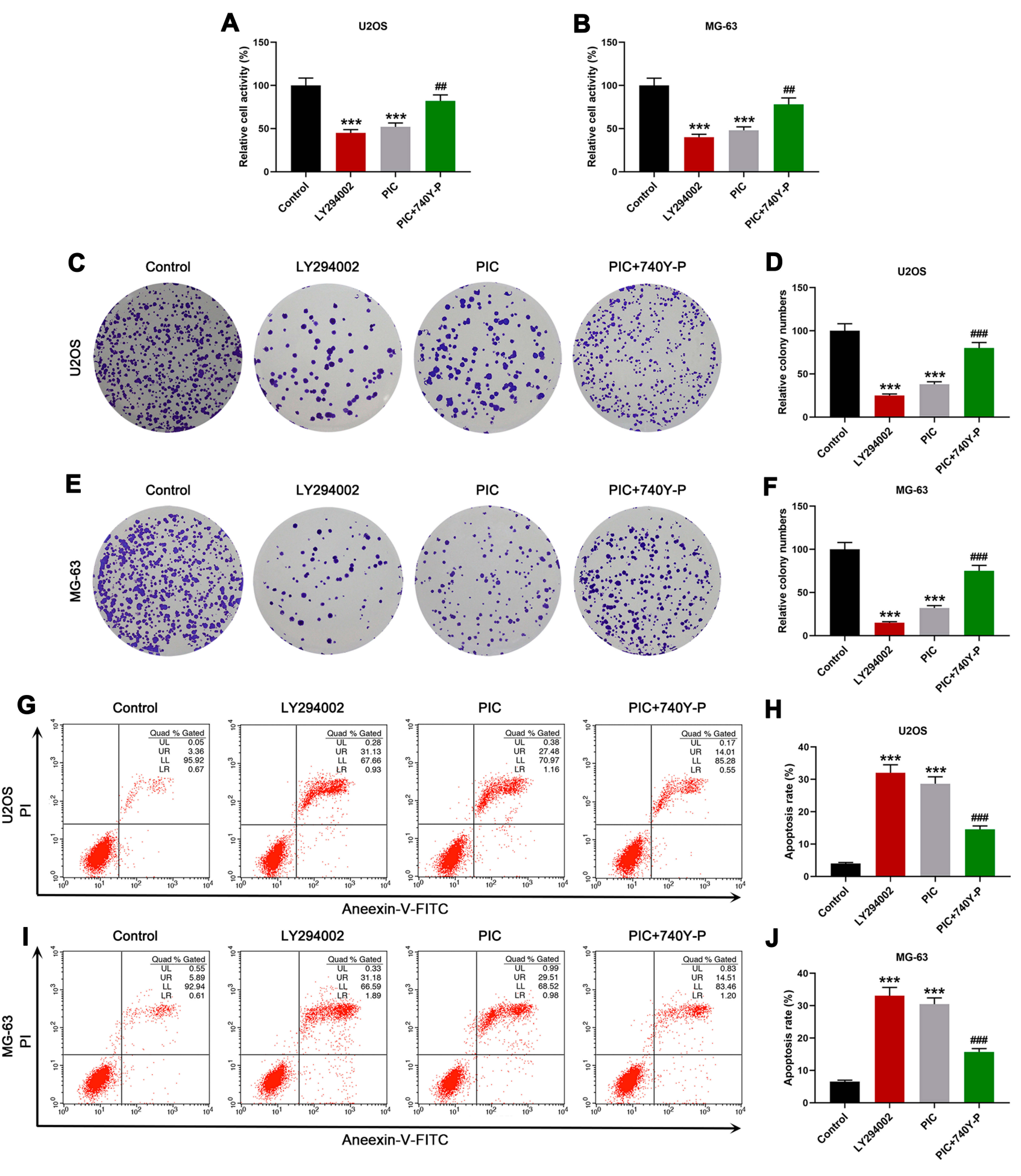

Figure 4 PI3K/AKT/mTOR pathway agonist rescued the effects of piceatannol (PIC) on osteosarcoma cells. In this figure, human osteosarcoma cell lines (U2OS and MG-63) were treated with PI3K/AKT/mTOR pathway inhibitor (LY294002, $10 \mu \mathrm{mol} / \mathrm{L})$, activator (740Y-P, I5 $\mu \mathrm{mol} / \mathrm{L})$, or $740 \mathrm{Y}-\mathrm{P}$ combined with PIC (80 $\mu \mathrm{mol} / \mathrm{L})$, and untreated cells were served as controls. (A, B) The activities of human osteosarcoma cell lines (U2OS and MG-63) after treatment were detected by 3-(4,5-dimethythiazol-2-yl)2,5-diphenyltetrazolium bromide (MTT) assays. (C-F) The cloning images and quantitative analysis of treated human osteosarcoma cell lines (U2OS and MG-63) were measured by colony formation assay. (G-J) The apoptosis rates of human osteosarcoma cell lines (U2OS and MG-63) after treatment were determined though a flow analyzer. $* * * P<0.001$, vs Control; ${ }^{\#} P<0.01,{ }^{\# \#} P<0.001$, vs PIC. All experiments were implemented in triplicate.

apoptosis, thus preventing the further development of tumors. Among the related pathways, this study further disclosed that PIC restrained the expressions of PI3K/Akt/
mTOR pathway-related proteins, so we speculated that the anti-cancer effect of PIC on osteosarcoma might be realized through this pathway. In previous studies, it has been 

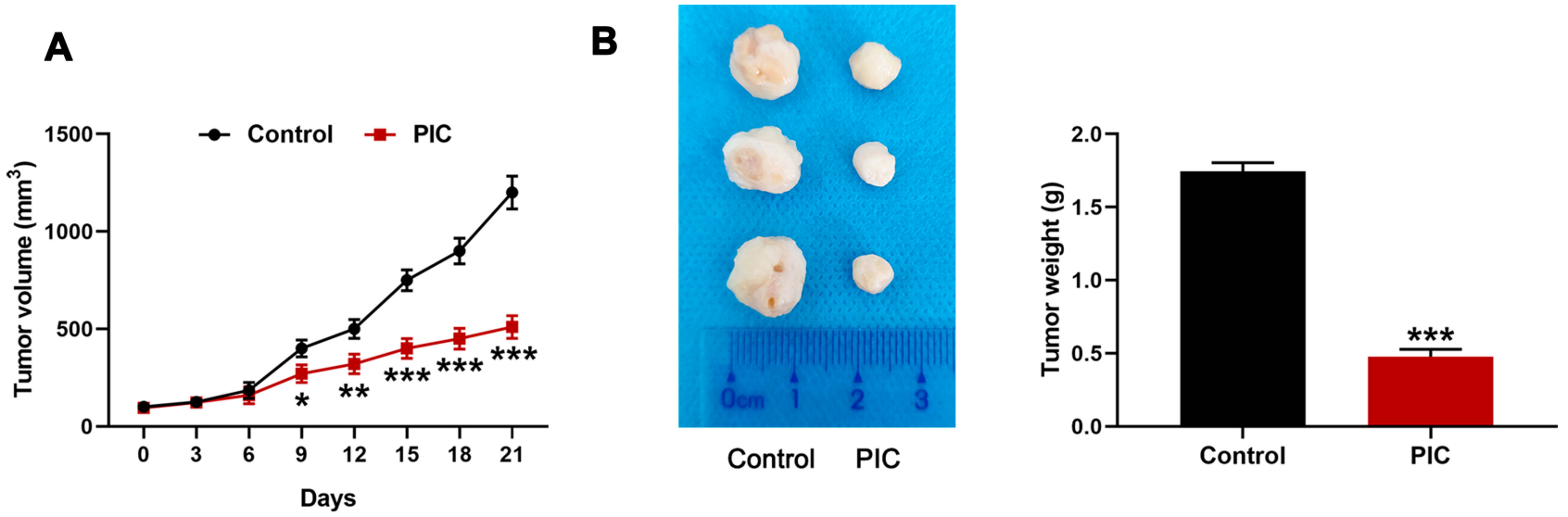

Figure 5 Piceatannol (PIC) restrained the growth of osteosarcoma tumors in xenograft models. In this figure, osteosarcoma cells (U2OS) were subcutaneously injected into BALB/c nude mice $(n=6)$ to establish in vivo xenograft models, and PIC $(20 \mathrm{mg} / \mathrm{kg} / \mathrm{day})$ was intraperitoneally injected into mice ( $\mathrm{n}=3)$ when the volume of tumors grew about $100 \mathrm{~mm}^{3}$, and another 3 mice treated with normal saline were used as controls. (A) The volume of tumor was detected by a caliper every 3 days until $2 \mathrm{I}$ days. (B) On the 2 Ist day, the tumors were separated from mice and then weighed. $* P<0.05, * * P<0.01$, $* * * P<0.00$ I, vs Control.
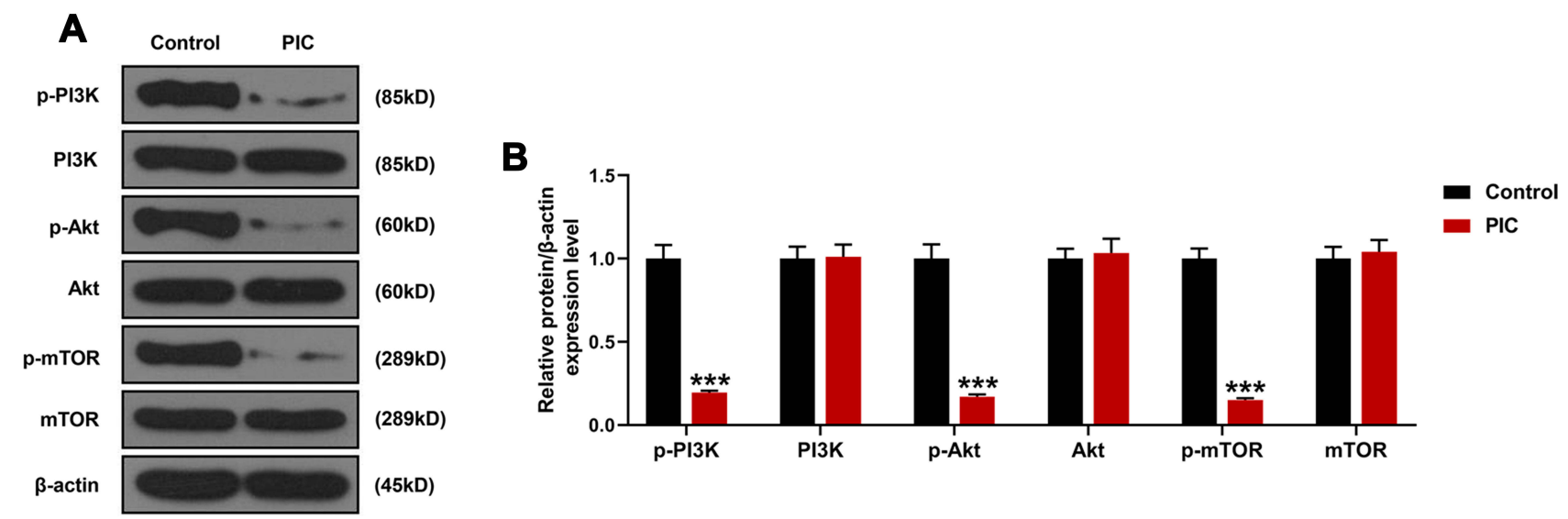

Figure 6 Piceatannol (PIC) suppressed the activation of PI3K/AKT/mTOR pathway in xenograft models. In this figure, osteosarcoma cells (U2OS) were subcutaneously injected into BALB/c nude mice $(n=6)$ to establish in vivo xenograft models, and PIC $(20 \mathrm{mg} / \mathrm{kg} /$ day $)$ was intraperitoneally injected into mice ( $\mathrm{n}=3)$ when the volume of tumors grew about $100 \mathrm{~mm}^{3}$, and another three mice treated with normal saline were used as controls. (A-B) Western blot (WB) assay was implemented to measure the expression levels of phosphorylated $(\mathrm{P})$ or not $\mathrm{PI} 3 \mathrm{~K}, \mathrm{Akt}$ and $\mathrm{mTOR}$ in xenograft models after treatment. $* * * \mathrm{P}<0.00 \mathrm{I}$, vs Control. All experiments were implemented in triplicate.

proved that the disordered $\mathrm{PI} 3 \mathrm{~K} / \mathrm{AKT} / \mathrm{mTOR}$ signaling was closely related to the proliferation and growth of osteosarcoma. ${ }^{23}$ In other plant ingredients, Li et $\mathrm{al}^{24}$ have demonstrated that honokiol could reduce the protein expression levels of PI3K, p-Akt and p-mTOR in osteosarcoma cells, thereby inducing autophagy and apoptosis of tumor cells. Wang et $\mathrm{al}^{25}$ reported that cucurbitacin E restricted the proliferation and invasion of osteosarcoma cells, induced cell cycle arrest, and promoted apoptosis through the weakening of $\mathrm{PI} 3 \mathrm{~K} / \mathrm{AKT} / \mathrm{mTOR}$ signaling pathway. These findings supported that the inactivation of the PI3K/AKT/mTOR signaling pathway had inhibitory effect on the progression of osteosarcoma cells.

$\mathrm{PI} 3 \mathrm{~K} / \mathrm{AKT} / \mathrm{mTOR}$ is identified as one of the major signaling pathways in cancer, which was directly concerned with cellular proliferation, exercise, cell cycle and tumour progression. ${ }^{26,27}$ In some diseases, the activation of this pathway can decrease apoptosis, allowing diffusion, and accelerate metastasis. ${ }^{28}$ In order to further verify the anti-tumor activity of PIC on osteosarcoma was realized through $\mathrm{PI} 3 \mathrm{~K} / \mathrm{AKT} / \mathrm{mTOR}$ signal transduction, this study used this pathway inhibitor and activator to the treat osteosarcoma cells. As for the experimental analysis results, we acquired that the effects of PIC on osteosarcoma cells were consistent with the activity of pathway inhibitor, whereas pathway activator could eliminate the inhibitory action of PIC on cell activity and proliferation, as well as alleviate cell apoptosis. In other cell studies, Wang et $\mathrm{al}^{29}$ showed that PIC could protect heart cells from oxidative stress injury by modulating the PI3K/Akt/ 
eNOS signaling pathway. Moreover, Song et $\mathrm{al}^{30}$ showed that PIC could restrain the activation of PI3K and inhibit the metastasis potential of human breast epithelial cells, as well as its effect was better than resveratrol. Based on the above in vitro cell experiments, it could be concluded that the proliferation suppression and apoptosis induction functions of PIC in osteosarcoma cells might be achieved by inactivating the $\mathrm{PI} 3 \mathrm{~K} / \mathrm{AKT} / \mathrm{mTOR}$ signaling pathway.

In previous animal studies, researches on PIC mainly focused on metabolic diseases. ${ }^{31}$ For example, UchidaMaruki et $\mathrm{al}^{32}$ applied PIC to mice on high-fat diet, and found that PIC not only did not affect visceral fat gain or weight gain in mice, but also reduced the fasting glucose level of mice, suggesting that PIC might have application value in the prevention of diabetes. In addition, PIC has been reported to inhibit the invasion of hepatocellular carcinoma cell by scavenging intracellular reactive oxygen species, and restrain the growth and metastasis of solid tumors in mouse models with hepatocellular carcinoma. ${ }^{33}$ For in-depth exploration, this study further constructed xenograft mouse models by subcutaneous injection of osteosarcoma cells, and treated them with PIC. The observation results indicated that although the tumor growth of the PIC-treated mice was increased with time, the trend was observably slower than that of the control mice from 9th day. In addition, this study also correspondingly verified that $\mathrm{PI} 3 \mathrm{~K} / \mathrm{AKT} / \mathrm{mTOR}$ signal pathway was arrested in tumor tissues of xenograft mouse models treated with PIC, which again confirmed that PIC could limit the growth of osteosarcoma through this pathway in vivo.

\section{Conclusions}

In conclusion, PIC suppressed the proliferation and induced apoptosis of osteosarcoma cells, as well as limited the growth of in vivo tumors through regulating PI3K/ AKT/mTOR pathway. These findings in this study might provide research basis for PIC in the treatment of osteosarcoma, which is expected to be the future therapeutic of this disease.

\section{List of Abbreviations}

PIC, piceatannol; WB, Western blot; PBS, phosphate buffer saline; PVDF, polyvinylidene fluoride; SDSPAGE, sodium dodecyl sulfate-polyacrylamide gel electrophoresis; SD, standard deviation; ANOVA, analysis of variance.

\section{Availability of Data and Materials}

The analyzed data sets generated during the study are available from the corresponding author on reasonable request.

\section{Ethics and Consent Statement}

All animal experiments involved in this study were authorized by the Ethic Committee of Shengjing Hospital of China Medical University, and followed the guidelines of Animal Care and Institutional Ethical in China. No human is involved in this research.

\section{Disclosure}

The authors declare no conflicts of interest in this work.

\section{References}

1. Mirabello L, Troisi RJ, Savage SA. Osteosarcoma incidence and survival rates from 1973 to 2004: data from the surveillance, epidemiology, and end results program. Cancer. 2009;115(7):1531-1543. doi:10.1002/cncr.24121

2. Meazza C, Scanagatta P. Metastatic osteosarcoma: a challenging multidisciplinary treatment. Expert Rev Anticancer Ther. 2016;16 (5):543-556. doi:10.1586/14737140.2016.1168697

3. Harrison DJ, Geller DS, Gill JD, Lewis VO, Gorlick R. Current and future therapeutic approaches for osteosarcoma. Expert Rev Anticancer Ther. 2018;18(1):39-50. doi:10.1080/14737140.2018. 1413939

4. Friebele JC, Peck J, Pan X, Abdel-Rasoul M, Mayerson JL. Osteosarcoma: a meta-analysis and review of the literature. $\mathrm{Am}$ J Orthoped. 2015;44(12):547-553.

5. Wang W, Yang J, Wang Y, et al. Survival and prognostic factors in Chinese patients with osteosarcoma: 13-year experience in 365 patients treated at a single institution. Pathol Res Pract. 2017;213 (2):119-125. doi:10.1016/j.prp.2016.11.009

6. Kager L, Tamamyan G, Bielack S. Novel insights and therapeutic interventions for pediatric osteosarcoma. Future Oncol. 2017;13 (4):357-368. doi:10.2217/fon-2016-0261

7. Ferrari S, Bielack SS, Smeland S, et al. EURO-B.O.S.S.: a European study on chemotherapy in bone-sarcoma patients aged over 40: outcome in primary high-grade osteosarcoma. Tumori. 2018;104 (1):30-36. doi:10.5301/tj.5000696

8. Piotrowska H, Kucinska M, Murias M. Biological activity of piceatannol: leaving the shadow of resveratrol. Mutat Res. 2012;750 (1):60-82. doi:10.1016/j.mrrev.2011.11.001

9. Rossi M, Caruso F, Antonioletti R, et al. Scavenging of hydroxyl radical by resveratrol and related natural stilbenes after hydrogen peroxide attack on DNA. Chem Biol Interact. 2013;206(2):175-185. doi:10.1016/j.cbi.2013.09.013

10. Aayush K, Samarth T, Amit M, Archana T. Piceatannol: a potential futuristic natural stilbene as fetal haemoglobin inducer. $J$ Clin Diagnostic Res. 2013;7(12):3028-3031.

11. Lee HH, Park SA, Almazari I, Kim EH, Na HK, Surh YJ. Piceatannol induces heme oxygenase-1 expression in human mammary epithelial cells through activation of ARE-driven Nrf2 signaling. Arch Biochem Biophys. 2010;501(1):142-150. doi:10.1016/j.abb.2010.06.011

12. Seyed MA, Jantan I, Bukhari SN, Vijayaraghavan K. A comprehensive review on the chemotherapeutic potential of piceatannol for cancer treatment, with mechanistic insights. J Agric Food Chem. 2016;64 (4):725-737. doi:10.1021/acs.jafc.5b05993 
13. Du M, Zhang Z, Gao T. Piceatannol induced apoptosis through up-regulation of microRNA-181a in melanoma cells. Biol Res. 2017;50(1):36. doi:10.1186/s40659-017-0141-8

14. Hsieh TC, Lin CY, Lin HY, Wu JM. AKT/mTOR as novel targets of polyphenol piceatannol possibly contributing to inhibition of proliferation of cultured prostate cancer cells. ISRN Urol. 2012;2012:272697. doi:10.5402/2012/272697

15. Mori K, Blanchard F, Charrier C, et al. Conditioned media from mouse osteosarcoma cells promote MC3T3-E1 cell proliferation using JAKs and PI3-K/Akt signal crosstalk. Cancer Sci. 2008;99 (11):2170-2176. doi:10.1111/j.1349-7006.2008.00919.x

16. Chang JK, Hsu YL, Teng IC, Kuo PL. Piceatannol stimulates osteoblast differentiation that may be mediated by increased bone morphogenetic protein-2 production. Eur J Pharmacol. 2006;551 (1-3):1-9. doi:10.1016/j.ejphar.2006.08.073

17. Li Z, Ma R, Khan M, Liu C, Cui X, Li Y. Piceatannol inhibits akt activation, induces $\mathrm{G} 2 / \mathrm{M}$ phase arrest and mitochondrial apoptosis and augments cisplatin efficacy in u2os osteosarcoma cells. Pak J Zool. 2019;51(6):2337-2344. doi:10.17582/journal.pjz/2019.51.6.2337.2344

18. Les F, Deleruyelle S, Cassagnes LE, et al. Piceatannol and resveratrol share inhibitory effects on hydrogen peroxide release, monoamine oxidase and lipogenic activities in adipose tissue, but differ in their antilipolytic properties. Chem Biol Interact. 2016;258:115-125. doi:10.1016/j.cbi.2016.07.014

19. Kapoor S. Tumor growth limiting effects of piceatannol. Biomed Res Int. 2013;2013:514349. doi:10.1155/2013/514349

20. Ko HS, Lee HJ, Kim SH, Lee EO. Piceatannol suppresses breast cancer cell invasion through the inhibition of MMP-9: involvement of PI3K/AKT and NF-kappaB pathways. J Agric Food Chem. 2012;60(16):4083-4089. doi:10.1021/jf205171g

21. Yan L, Lu L, Hu F, Shetti D, Wei K. Piceatannol attenuates RANKL-induced osteoclast differentiation and bone resorption by suppressing MAPK, NF-kappaB and AKT signalling pathways and promotes Caspase3-mediated apoptosis of mature osteoclasts. Royal Society Open Sci. 2019;6(6):190360. doi:10.1098/rsos.190360

22. Wolter F, Clausnitzer A, Akoglu B, Stein J. Piceatannol, a natural analog of resveratrol, inhibits progression through the $\mathrm{S}$ phase of the cell cycle in colorectal cancer cell lines. $J$ Nutr. 2002;132 (2):298-302. doi:10.1093/jn/132.2.298
23. Keremu A, Maimaiti X, Aimaiti A, et al. NRSN2 promotes osteosarcoma cell proliferation and growth through PI3K/Akt/mTOR and Wnt/beta-catenin signaling. Am J Cancer Res. 2017;7(3):565-573.

24. Li Z, Dong H, Li M, et al. Honokiol induces autophagy and apoptosis of osteosarcoma through PI3K/Akt/mTOR signaling pathway. Mol Med Rep. 2018;17(2):2719-2723. doi:10.3892/mmr.2017.8123

25. Wang Y, Xu S, Wu Y, Zhang J. Cucurbitacin E inhibits osteosarcoma cells proliferation and invasion through attenuation of PI3K/AKT/ mTOR signalling pathway. Biosci Rep. 2016;36:6. doi:10.1042/ BSR20160165

26. Dey N, De P, Leyland-Jones B. PI3K-AKT-mTOR inhibitors in breast cancers: from tumor cell signaling to clinical trials. Pharmacol Ther. 2017;175:91-106. doi:10.1016/j.pharmthera.2017.02.037

27. Edlind MP, Hsieh AC. PI3K-AKT-mTOR signaling in prostate cancer progression and androgen deprivation therapy resistance. Asian J Androl. 2014;16(3):378-386. doi:10.4103/1008-682X.122876

28. Pierobon M, Ramos C, Wong S, et al. Enrichment of PI3K-AKTmTOR pathway activation in hepatic metastases from breast cancer. Clin Cancer Res. 2017;23(16):4919-4928. doi:10.1158/1078-0432. CCR-16-2656

29. Wang D, Zhang Y, Zhang C, Gao L, Li J. Piceatannol pretreatment alleviates acute cardiac injury via regulating PI3K-Akt-eNOS signaling in H9c2 cells. Biomed Pharmacother. 2019;109:886-891. doi:10.1016/j.biopha.2018.10.120

30. Song NR, Hwang MK, Heo YS, Lee KW, Lee HJ. Piceatannol suppresses the metastatic potential of MCF10A human breast epithelial cells harboring mutated H-ras by inhibiting MMP-2 expression. Int J Mol Med. 2013;32(4):775-784. doi:10.3892/ijmm.2013.1449

31. Kershaw J, Kim KH. The therapeutic potential of piceatannol, a natural stilbene, in metabolic diseases: a review. J Med Food. 2017;20(5):427-438. doi:10.1089/jmf.2017.3916

32. Uchida-Maruki $H$, Inagaki $H$, Ito $R$, Kurita I, Sai M, Ito $T$. Piceatannol lowers the blood glucose level in diabetic mice. Biol Pharm Bull. 2015;38(4):629-633. doi:10.1248/bpb.b15-00009

33. Kita Y, Miura Y, Yagasaki K. Antiproliferative and anti-invasive effect of piceatannol, a polyphenol present in grapes and wine, against hepatoma AH109A cells. J Biomed Biotechnol. 2012;2012:672416. doi:10.1155/2012/672416

\section{Publish your work in this journal}

Cancer Management and Research is an international, peer-reviewed open access journal focusing on cancer research and the optimal use of preventative and integrated treatment interventions to achieve improved outcomes, enhanced survival and quality of life for the cancer patient.
The manuscript management system is completely online and includes a very quick and fair peer-review system, which is all easy to use. Visit http://www.dovepress.com/testimonials.php to read real quotes from published authors. 